\title{
Reproductive coercion and abuse against women from minority ethnic backgrounds: Views of service providers in Australia
}

\author{
Laura Tarzia*a, Heather Douglas ${ }^{b}$, Nicola Sheeranc \\ Department of General Practice, The University of Melbourne, Melbourne, Victoria, Australia ; School of \\ Law, University of Queensland, Saint Lucia, Queensland, Australiab; School of Applied Psychology, \\ Griffith University, Nathan, Queensland, Australiac
}

*Corresponding Author: Laura Tarzia Email: laura.tarzia@unimelb.edu.au

\begin{abstract}
Reproductive coercion and abuse is defined as behaviour that deliberately interferes with a person's reproductive autonomy. It is typically perpetrated by men against women in a context of fear and control and includes forcing a woman to become pregnant or to terminate a pregnancy. There is a dearth of qualitative research investigating experiences of reproductive coercion and abuse, particularly for women from minority ethnic backgrounds. In this study, we address this gap through qualitative analysis of data from six focus groups with service providers in Australia. Three main themes were developed: 1) exploiting structural inequalities; 2) women as reproductive property; and 3) pregnancy as a form of control. Findings confirm the complex interplay between reproductive coercion and abuse, intimate partner and sexual violence, and suggest that community attitudes towards women's role in sex and reproduction and structural risk factors may complicate this nexus even further for minority ethnic women. It is important for service providers supporting minority ethnic women - particularly in antenatal and abortion care - to be aware of reproductive coercion and abuse. Similarly, policies concerning access to financial support for minority ethnic women should acknowledge the critical role this could play in facilitating or preventing abuse.
\end{abstract}

Keywords: reproductive coercion, violence against women, migrant women, ethnic minorities, Australia

\section{Introduction}

Reproductive coercion and abuse is a complex and under-studied form of violence against women. Often referred to as simply "reproductive coercion", it has been defined as "any attempt to dictate a woman's reproductive choices or interfere with her reproductive autonomy" (Miller et al. 2010). We prefer the term "reproductive coercion and abuse", since it foregrounds the context of fear and control that typically accompanies this behaviour, as well as acknowledges the use of overt physical or sexual tactics as well as coercive ones (Tarzia et al. 2018). Behaviours considered to be types of reproductive coercion and abuse include: coercing a woman to become or remain pregnant against her will; tampering with birth control in order to promote pregnancy; and pressuring or forcing a woman to undergo a termination (Grace and Anderson 2018). Although robust research on the prevalence and 
impacts of reproductive coercion and abuse is lacking (Tarzia et al. 2018; Rowlands and Walker 2019) and conceptual inconsistencies render measurement challenging (Tarzia et al. 2018), limited data suggests that reproductive coercion and abuse is both common (Rowlands and Walker 2019) and harmful to women's mental and physical health (McCauley et al. 2014; Kazmerski et al. 2015).

Putting aside the definitional and measurement issues, existing research does consistently suggest that reproductive coercion and abuse has strong associations with other forms of intimate partner violence and sexual violence (Grace and Anderson 2018; Basile et al. 2018; Sharman et al. 2018; Price et al. 2019; Katz et al. 2017). A recent Australian study, for example, suggested that over $20 \%$ of women accessing pregnancy counselling service, Children By Choice, because of reproductive coercion and abuse had also experienced intimate partner violence (Price et al. 2019). What is unclear, however, are the mechanisms, context and directionality of these relationships. Pregnancy may be a catalyst for an abusive partner or family member to commence or escalate physical or psychological violence (Brownridge et al. 2011) or alternatively, physical, sexual or psychological violence may be a mechanism through which reproductive coercion and abuse is enacted. Both pathways are plausible in the context of an interpersonal relationships characterised by fear and control, yet existing qualitative research does not illuminate these nuances to a large extent.

A further critical gap in the knowledge base lies around the experiences of women from minority ethnic backgrounds. Reproductive coercion and abuse research to date has predominantly focused on mainstream populations in high-income countries such as the USA. Yet, from the broader literature on intimate partner violence, we know that structural inequalities and patriarchal gender norms can make minority ethnic women more susceptible to harm, and less able to access support (Childress 2013; Vaughan et al. 2016; Ayyub 2000; Ahmad et al. 2009; Ahmad et al. 2004; Nikolajski et al. 2015; Maher and Segrave 2018; Balice et al. 2019; Do, Weiss and Pollack 2013). This is particularly the case for minority ethnic women who are migrants (Maher and Segrave 2018). Research suggests that these women may face discrimination, lack of knowledge about services, migration stress, language barriers and visa insecurity (Allimant and Ostapiej-Piatkowski 2011; Maher and Segrave 2018; Forbes-Mewett and McCulloch 2015), which can compound the risks associated with intimate partner violence. However, very little is known about how these intersectional risk factors play out in the specific context of reproductive coercion and abuse , where the added layer of community norms around reproduction may also contribute (Hawkey, Ussher and Perz 2018).

This paper addresses the abovementioned gap in the literature by exploring service provider perspectives of migrant minority ethnic women's experiences of reproductive coercion and abuse in Australia. We draw on focus groups with specialist women's service providers in the states of Queensland and Victoria to identify how reproductive coercion and abuse is enacted against migrant minority ethnic women and its intersections with intimate partner and sexual violence. Our guiding research question was "What does reproductive coercion and abuse look like for women from migrant minority ethnic backgrounds in Australia?"

We use the term "migrant minority ethnic women" to describe a particular cohort of women who are from minority ethnic backgrounds and who have arrived in Australia either via traditional immigration pathways or as asylum seekers. This is to distinguish them from 
minority ethnic women born in Australia, and from immigrant women from countries such as the USA, the UK and Ireland, South Africa, Canada and New Zealand, although some of the same issues may also be faced by members of these groups. As of 2018, almost $30 \%$ of the Australian population was born overseas, (Australian Bureau of Statistics 2016, 2020). Although many of these hail from the UK and Ireland, a growing proportion come from countries such as China, India, Vietnam, Italy and Sri Lanka, with growing representation from Middle-Eastern and African countries such as Sudan (the latter particularly in the state of Victoria) (Australian Bureau of Statistics 2020). It is important to acknowledge that there are a diverse range of cultural views and groupings within each of these countries. Furthermore, it is not our intention to suggest that "culture" is a fixed or innate feature for any individual or group of individuals (AMES Australia and Australian Government Department of Social Services 2016). However, what almost all cultures have in common to a greater or lesser extent are types of patriarchal beliefs associated with violence against women (Do, Weiss and Pollack 2013; Balice et al. 2019; Webster et al. 2018; Fakunmoju and Bammeke 2017). These beliefs - in whatever manifestation they take in a particular community - and how they intersect with structural, societal, and relationship issues, is the focus of this paper.

\section{Materials and Methods}

This paper draws on data collected between December 2019 and January 2020 as part of a broader project exploring the views of specialist domestic violence and women's service providers on supporting women experiencing reproductive coercion and abuse. Specifically, the project involved services offering legal advice and information to women experiencing violence, since we were interested in the challenges these services might face relating to how reproductive coercion and abuse is addressed (or not) in the legal context. We did not initially set out to explore the particular issues faced by migrant minority ethnic women, however, many of the service providers spontaneously chose to narrate experiences and anecdotes that related to this topic, which we have analysed here.

We undertook six focus groups with specialist women's services in the states of Victoria and Queensland. These states were chosen because they have similar legislation and policies on domestic violence. Participating services included a women's legal service; a domestic violence service specialising in supporting women from minority ethnic backgrounds; and a general domestic violence support service in each state. Thirty-eight staff members took part in six focus groups across these services. Eleven of the participants worked as lawyers, one worked as a para-legal, and 26 worked as social workers or counsellors. All of the participants provided support to women who have experienced intimate partner violence. A breakdown of participant distribution across the services is provided in Table 1. All participants in the study were female, which reflects the demographic profile of workers in this area. Although we did not collect information about participants' cultural identity, the two services for minority ethnic women were primarily staffed by workers who were themselves from minority ethnic backgrounds. 
Tarzia et al. Accepted Manuscript

Culture, Health \& Sexuality

Published version available at https://doi.org/10.1080/13691058.2020.1859617

\begin{tabular}{|l|l|l|l|l|}
\hline FG \# & State & Type of Service & $\begin{array}{l}\text { Number of } \\
\text { participants }\end{array}$ & $\begin{array}{l}\text { Type } \\
\text { practitioners }\end{array}$ \\
\hline 1 & QLD & Domestic violence (general) & 7 & $\begin{array}{l}\text { Counsellors/social } \\
\text { workers }\end{array}$ \\
\hline 2 & QLD & Legal & 5 & $\begin{array}{l}\text { Lawyers/social } \\
\text { workers }\end{array}$ \\
\hline 3 & VIC & Legal & 9 & $\begin{array}{l}\text { Lawyers/social } \\
\text { workers }\end{array}$ \\
\hline 4 & QLD & Domestic violence (minority ethnic) & 6 & $\begin{array}{l}\text { Counsellors/social } \\
\text { workers }\end{array}$ \\
\hline 5 & VIC & Domestic violence (minority ethnic) & 5 & $\begin{array}{l}\text { Counsellors/social } \\
\text { workers }\end{array}$ \\
\hline 6 & VIC & Domestic violence (general) & 6 & $\begin{array}{l}\text { Counsellors/social } \\
\text { workers }\end{array}$ \\
\hline
\end{tabular}

Table 1. Focus group details

\section{Recruitment}

The research team chose services to approach for the study based on whether they provided legal advice and information to women experiencing intimate partner violence. We aimed for matched services in both states where possible (i.e. one legal service, one general domestic violence and one multicultural). The directors from each service were approached, sent information about the study and asked to contact the research team if they wished to take part. After speaking with the researchers and confirming their interest in participating, directors were asked to invite staff in their service to be involved in a focus group at a convenient date and time. Copies of a plain language description of the study were made available for staff to read prior to attending. Staff were not obliged to attend the focus group if they did not wish to. Each staff member provided individual written consent before commencing the focus groups.

\section{Data collection}

Focus groups were facilitated by HD and NS in Queensland and combinations of HD, NS and LT in Victoria (one researcher facilitated and the other took notes and supported). We utilised a semi-structured set of questions which allowed some flexibility, whilst also ensuring that we covered all the necessary topics during the limited time staff had available. Each focus group ran for approximately 60-90 minutes and was audio-recorded and transcribed by a professional transcriber.

\section{Data Analysis}

We used Braun and Clarke's (2019) reflexive thematic analysis to help make sense of the focus group data. All authors read the transcripts multiple times and discussed initial ideas and potential themes collaboratively. Author LT then re-read the transcripts in more detail and developed initial descriptive codes. The second step involved interpretive coding where descriptive codes were grouped together and underlying meanings teased out. This process was repeated to develop overarching themes that describe reproductive coercion and abuse for migrant minority ethnic women. These were reviewed by HD and NS. 
A key characteristic of Braun and Clarke's approach - as is suggested by its name - is its focus on researcher reflexivity. Reflexive thematic analysis accepts the subjectivity of qualitative methods and the interplay between the researcher/'s position and interpretation of the data. Although we drew on an intersectional feminist theoretical framework (Lykke 2010) to help foreground how multiple levels of oppression (race, gender, immigrant status) and constraining normativities might impact upon the experiences of migrant minority ethnic women, all the researchers are white and Australian-born, which is very likely to have influenced our interpretation of the data despite our best efforts. We would therefore welcome continued discussion with our minority ethnic colleagues and others with lived experience in this area in order to gain a more nuanced perspective on the issue of reproductive coercion and abuse.

We aimed to maximise trustworthiness by engaging all authors in the analytic process and ensuring collaborative discussion about how we were interpreting the data and how our respective positions might be influencing this. We encouraged consistency in how data were collected due to the fact that researcher NS was present at all focus groups and have aimed for transparency in how our methods have been reported here.

\section{Ethical issues}

Confidentiality of the participants' names and employers was ensured by the researchers. References to comments made in the focus groups are identified by a number given to the focus group transcript (e.g. FG1). Ethical approval for the study was granted by Griffith University human ethics committee (approval number 2017000964).

\section{Results}

Three main themes are outlined below. These describe the unique context of reproductive coercion and abuse for women from migrant minority ethnic backgrounds as perceived by service providers. We conclude with a case study outlined by one of the participants. This highlights how some migrant minority ethnic women can experience all of these harms concurrently.

\section{Exploiting structural risk factors}

A major theme within the data was that women from migrant minority ethnic backgrounds could be profoundly vulnerable to reproductive coercion and abuse due to a range of structural factors. In particular, the threat of visa cancellation if a woman did not comply with the perpetrator's reproductive demands was a key issue that many women faced. Many migrant minority ethnic women arrive in Australia on a partner visa which leads to uncertainty around their immigration status. Participants described how perpetrators exploited this uncertainty - either overtly or in more subtle ways - in order to force women into engaging in unprotected sex, keeping or terminating a pregnancy.

It's not like the partner's forcing them [to have sex], physically and all that, but if she says no, then she's got to deal with lots of other problems and because of pressure of financial, immigration, just somewhere to stay, lots of other things, they will engage in sex just because of that. (FG 4) 
Participant 1: If you decide to keep the baby, I'm going to cancel it [visa]. I'm going to...

Participant 2: That sort of thing.

Participant 3: We hear that a lot.

Participant 2: If you're going to go through with the pregnancy, I'm going to withdraw [support].

Participant 1: We're going to send you and your daughter back home. (FG 5)

I'll cancel your visa... the clients are incredibly vulnerable to that threat, and the immigration department is... The moment that the other party says, it wasn't my child, the immigration department puts the responsibility on the woman to prove the paternity of the child, no presumptions apply. So, they require paternity testing, and if not, a declaration from the court, which is really disgusting for all kinds of reasons. (FG 3)

Related to this was the fact that many migrant minority ethnic women were financially dependent on the perpetrator. Some women were not able to work due to the requirements of their visas or did not have the right to access government supports. This placed women in an incredibly difficult situation if they went against the perpetrator's wishes with regards to a pregnancy, since they were left with few options to support themselves or a new baby.

The [perpetrator] withdrew all the financial support and everything so leaving the woman pregnant with no support, nowhere to go. (FG 5)

Participants also described situations where perpetrators exploited women's lack of knowledge about Australian culture and their legal rights. In some cases, perpetrators were even able to access a woman's electronic health record to keep track of her medical appointments and ensure that she was not seeking pregnancy care or termination. One service provider recounted how women could be told by an Australian partner that the sexual abuse they were experiencing was normal:

When sexual abuse was discussed within the session, it was kind of like an eye opened up for [her]. Saying, "Oh, I thought that because my husband is Australian, that's the way it should be." (FG 4)

\section{Women as reproductive property}

This theme reflected the belief that women from migrant minority ethnic backgrounds can be adversely affected by gendered community norms around women's role within a relationship and the broader family context. Participants felt that some migrant communities placed expectations on women to reproduce, with husbands, family members or the broader community taking 'ownership' over women's reproductive capacity. 
Culturally, it might be that their understanding is the role of the man to make certain decisions in the relationship, including when to have children and not, and it's the role of the woman to breed, essentially, and just have children. (FG 3)

The woman had no space in family planning whatsoever. Every time you see her she's pregnant, and the baby is still crawling, but she is pregnant again and it wasn't just the perpetrator who is the issue but the family - his family members also thought they had to have that...It was their income source. (FG 5)

So, the mum [is] actually involve[ed] in the sexual activity to make sure that the woman [is] actually pregnant... She's actually attending in the room! (FG 5)

The centrality of women's reproductive role in some communities meant that pregnancy could also be used as justification for a perpetrator to abuse a woman physically or psychologically. Participants described examples where women were subjected to other forms of violence for 'failing' to become pregnant, or for choosing to remain pregnant when the partner wanted her to terminate. The sex of the unborn child (primarily son preference (Edvardsson et al. 2018)) could similarly be a catalyst for reproductive coercion and abuse and other forms of co-occurring violence:

They [couple] weren't able to conceive, and so he [husband] blamed her for that...That was the excuse, or reason, or whatever, for him to perpetrate family violence, and then to seek other women...Because she wasn't performing her 'functions'. (FG 3)

She clearly mentioned to me there was some form of control and abuse already happening in the relationship. A part of that was because she was pregnant with a female child, she was asked to terminate the pregnancy and, as she refused it, it also escalated abuse from her partner as well as from her in-laws. (FG 5)

Participants also described cultural or religious beliefs about abortion, with many indicating that some groups were strongly against the practice, even when the woman had been raped. This was particularly an issue for women from collectivist cultures, where favourable social self-worth is judged by members of the community (Do, Weiss, and Pollack 2013).

I had a client, a Vietnamese client, who was raped. She never intended to have a relationship with that man. But the community would not allow her - her friends and the people in the community would not allow her to have a termination. (FG 1)

There's a cultural expectation that you don't have an abortion. If you get pregnant, that's it, you're having a baby, you're seeing that pregnancy through. (FG 2) 
Linked to community beliefs about women's reproductive capacity was the lack of sexual autonomy that service providers often saw in migrant minority ethnic women who had experienced reproductive coercion and abuse. Women often did not have the right to control the timing and frequency of sexual intercourse, nor the option to make choices about contraception. For instance, a practitioner from one of the multicultural services described:

We hear a lot from clients, that they didn't feel okay to say, let's use condoms or a contraceptive pill. Sometimes a perpetrator [is] directly telling [a] woman, I will need you to sex [sic] with me until we have a baby. (FG 4)

In some communities, having sex with one's husband was considered to be a "wifely duty"; women consequently did not feel that it was permissible to refuse, even when the sex was likely to result in pregnancy.

It's expected she has sex and she's owned by him and she has no say. (FG 2)

These demands for sex were not just perpetrated by men from within women's own communities. Participants from one of the multicultural services described a common scenario where Australian men would sponsor women from other countries (primarily countries such as Thailand and China) to come to Australia and then expect them to undertake unwanted sexual acts in return.

... So, they [perpetrators] make lots of comments like, you're here in Australia, beautiful country, because I brought you here and you need to - it is your responsibility to make me happy, whatever I want. So, they [are] making woman feel like they're only tools for sexual intercourse. (FG4)

In this context, as the participants rightly pointed out during their focus group discussion, the abuse is not linked to the woman's culture but an undertone of misogyny within mainstream Australian culture (Flood 2015).

\section{Pregnancy as a mechanism of control}

Several participants highlighted the use of pregnancy as a mechanism for controlling women's freedom. Forcing them to constantly be pregnant and caring for small children effectively imprisoned women in the home and restricted their ability to obtain economic independence. Furthermore, it ensured that a woman could not leave her partner, even when he was abusive towards her. In this sense, reproductive coercion and abuse in the form of pregnancy coercion can be seen as being linked to coercive control:

She was pregnant for literally 10 years. In my view, that really compromised her capacity to be independent. It compromised her capacity to really function in a self-determining way because she was so tired; either with very small children or being pregnant, or just having had a child for 10 years of her life. She said to me, "He does this to me to keep me in the relationship." (FG 2) 
Despite having had eight children...when we started seeing her, [she] was unable to actually conduct a transaction such as going to a shop and purchasing something. She was entirely isolated in the home as a result of having so many children. (FG 3)

On the other hand, in one focus group with a specialist service for minority ethnic women, there was a discussion around how denying women the opportunity to become pregnant was also a form of abuse, since it effectively prevented them from fulfilling the dream of having a family.

Our clients, when they marry someone and come here, or if they're married here, the thing is always to start a family, that's one of the important things for them. To settle down, have a family. But often...they are either forced to not have a child, or sometimes made - if there's a pregnancy, to have a termination. So, that really affects them emotionally and they've lost all the dreams of having the family (FG $4)$.

It was not clear from the focus groups why a perpetrator might attempt to force a woman to terminate a pregnancy, although as suggested by Douglas and Kerr (2018) it could be a way of ensuring that their own needs and wishes are prioritised in the relationship.

\section{Bringing the themes together}

The example below illustrates how all of the issues mentioned above can play out in practice for some migrant minority ethnic women. In it (which was a single case study reported by one of the practitioners), we see a combination of sexual assault, IPV, pregnancy preventing behaviour and the involvement of family members, creating a highly complex scenario that was challenging for service providers to address:

She was brought here by her husband and she was raped [by him], daily, from the moment that she arrived here. Then eventually she became pregnant. When [husband] realised that she was pregnant, he forced her to have a termination [because] the baby was forbidden, based on their religion. Then she went to her father-in-law to seek advocacy... I guess, to convince the son to keep the baby. But based on their religious background, she was within the time to have an abortion. So, how it happened was they made the appointment with the GP, but the person who made the appointment was her sister-in-law, who contacted the practice and provided her details and the husband's details... [The woman] approached the service, the GP asked her if she was sure about it, she became distraught and started crying. The GP asked for a moment to be with her, but in that moment, the husband approached her and whispered in her ear, "If you talk to the GP or confess whatever, I'm going to kill you..." So, she obviously couldn't say a word and the pregnancy was terminated. After that, she was 
advised to not have sexual intercourse, which was disregarded by the husband who continued assaulting her.

\section{Discussion}

This paper explored specialist service provider perceptions of reproductive coercion and abuse experienced by migrant minority ethnic women in Australia. Findings suggest that reproductive coercion and abuse for these women may occur at the intersection between patriarchal community norms specifically related to sex and reproduction, men's controlling and violent behaviours, and structural inequalities within Australian society. The paper contributes to a limited knowledge base around the dynamics and context of reproductive coercion and abuse and represents the first qualitative investigation into how it manifests for this sub-group of women in Australia (Grace et al. 2020; Nikolajski et al. 2015). Given that Australia is a multicultural society (Australian Bureau of Statistics 2020, 2016), it is critical that the experiences of migrant minority ethnic women be considered alongside those of their Australian-born peers in order to ensure that interventions and responses are appropriate to their needs.

Similar to findings from previous research with minority ethnic women (Maher and Segrave 2018; Raj and Silverman 2002; Douglas and Kerr 2018), service providers in this study identified the use of coercion on the part of perpetrators to exploit women's structural vulnerabilities such as visa status, financial insecurity and lack of knowledge about their legal rights in Australia. Existing research on migrant minority ethnic women's experiences of intimate partner violence strongly supports the use of immigration status as a mechanism by which to exert control over, and abuse women (Raj et al. 2005; Raj and Silverman 2002; Segrave 2017). However, this study confirms that immigration status can also be exploited for the specific purpose of interfering with migrant minority ethnic women's reproductive autonomy. Similarly, the threat of withdrawing financial support was recognised as a common strategy used by perpetrators to promote or prevent pregnancy. Although this tactic has also been described in the context of non-migrant women (Cappelletti, Gatimu and Shaw 2014), the ineligibility or inability of some women from migrant minority ethnic backgrounds to access government supports or intimate partner violence services (Douglas and Kerr 2018; Maher and Segrave 2018) places them in a particularly difficult position when their financial security is compromised.

Patriarchal community norms around sex and reproduction also play an important role in shaping some migrant minority ethnic women's experiences of reproductive coercion and abuse and how it intersects with intimate partner violence and sexual violence. Although studies have identified many of the ways that patriarchal norms can be exploited by perpetrators in the context of minority ethnic women's experiences of intimate partner violence more generally (Raj and Silverman 2002; Maher and Segrave 2018; Do, Weiss, and Pollack 2013; Balice et al. 2019), they have largely ignored the specific interaction between these norms and reproductive coercion and abuse. In particular, this study highlights how particular attitudes towards women's reproductive capacity can facilitate reproductive coercion and abuse. Hawkey, Ussher and Perz (2018), in their qualitative exploration of minority ethnic women's experiences of fertility control in Australia, reported similar findings about the role that culture can play in the context of reproduction. They describe how particular cultural or religious beliefs and patriarchal discourses can contribute to 
continuing gender inequities that impact on women's fertility (Hawkey, Ussher and Perz 2018). Women in their study described the expectation that they would become mothers, including pressure from husbands, family members, and the broader community. However, as recent research has highlighted (Tarzia et al. 2018), reproductive coercion and abuse goes beyond "pressure" or "expectations" and crosses a line into coercive and even physically violent behaviour. Thus, our study extends and builds upon Hawkey, Ussher and Perz's (2018) work, demonstrating that the "collective governance over women's reproductive bodies" (p.83) they describe can easily be exploited by perpetrators. If women did not fulfil their designated role or deviated from community expectations (either by falling pregnant, not falling pregnant or by having a baby of the "wrong" sex), this could be perceived as grounds for physical, sexual or psychological abuse to force them into a particular pregnancy outcome. (Edvardsson et al. 2018)

Similarly, beliefs about women's right to sexual autonomy were identified by participants as being related to reproductive coercion and abuse. Women from some migrant minority ethnic backgrounds were described as having little to no control over the timing or frequency of sexual intercourse, with sex seen as a "wifely duty". A few previous studies (Kasturirangan, Krishnan and Riger 2004; Abraham 1999; Ussher et al. 2017) with migrant women have highlighted the way that patriarchal discourses about women's sexual passivity and male sexual aggression can lead to unequal power relationships (Khoei, Whelan and Cohen 2008) and even normalise rape in marriage (Abraham 1999, 592); this was certainly the impression given by participants in our study. However, in general there is a dearth of research focusing on the sexual violence experiences of migrant minority ethnic women in relationships, with most of the literature examining the broader problem of sexual and reproductive health (Allotey et al. 2004; Mengesha et al. 2017). It is important to note here that we are not suggesting that minority ethnic women are the only ones to experience sexual entitlement from their male partners. Recent research into intimate partner sexual violence in women who were primarily Australian-born also found that perpetrators had similar ideas about being entitled to sex (Tarzia 2020). However, in the case of migrant minority ethnic women, the absence of sexual control can be compounded by a lack of contraceptive choice and religious or community objections to abortion, which may put them at repeated risk of unintended pregnancies (Ussher et al. 2017). Despite this, the connection between sexual violence and reproductive coercion and abuse is often overlooked (Tarzia et al. 2018), with most studies focusing only on connections between reproductive coercion and abuse and intimate partner violence (Grace and Anderson 2018). It is critical that future research engage with the issue of sexual violence in order to better understand the dynamics of reproductive coercion and abuse, particularly for minority ethnic women.

Lastly, some participants raised the use of reproductive coercion and abuse as a mechanism of control. Women could be forced to be continuously pregnant and caring for young children, which effectively imprisoned them in the home. The normalisation of large families in some minority ethnic communities such as those from Africa and the Middle East (Hawkey, Ussher and Perz 2018; Allotey et al. 2004; Ussher et al. 2012) may facilitate this form of abuse, although again large families are not necessarily unique to these women. Being constantly pregnant or responsible for young children potentially places women in a position where they are even more financially and emotionally compromised, which, in turn, exposes them to further exploitation by the perpetrator. Some participants also mentioned 
forced abortions as a way of denying women their desired role as a mother, but it is unclear how this might fit with the other behaviours; more research is recommended in this area.

Overall, our findings illuminate some of the more nuanced aspects of reproductive coercion and abuse for migrant minority ethnic women, in terms of how it intersects with intimate partner violence and sexual violence and the role of patriarchal community norms and structural inequalities as facilitators. Key findings from the study include the intersection between intimate partner sexual violence and reproductive coercion and abuse for migrant minority ethnic women, where a lack of sexual autonomy in relationships can combine with poor knowledge about, or access to, effective contraception. The intersection between visa insecurity and reproductive coercion and abuse was also a major theme in our data. The below diagram, based on one developed previously by Tarzia and colleagues (2018), demonstrates the intersections between reproductive coercion and abuse, intimate partner violence, sexual violence and family violence, with additional contributing factors that create an environment conducive to reproductive coercion and abuse. We have added both "patriarchal norms around sex and reproduction" and "structural inequalities" as contributing factors in the diagram.

\section{Contributing factors}

- Government policies that prevent or impede access to abortion or infringe on women's human rights

- Lack of access to abortion due to availability, eligibility etc.

- Negative social attitudes (towards abortion, childbirth, women's role)

- Lack of sexual health education

- Religious views that do not condone abortion

- Patriarchal norms around sex \& reproduction

- Lack of community knowledge about RCA

- Gender inequality or violencesupportive attitudes

- Structural inequalities

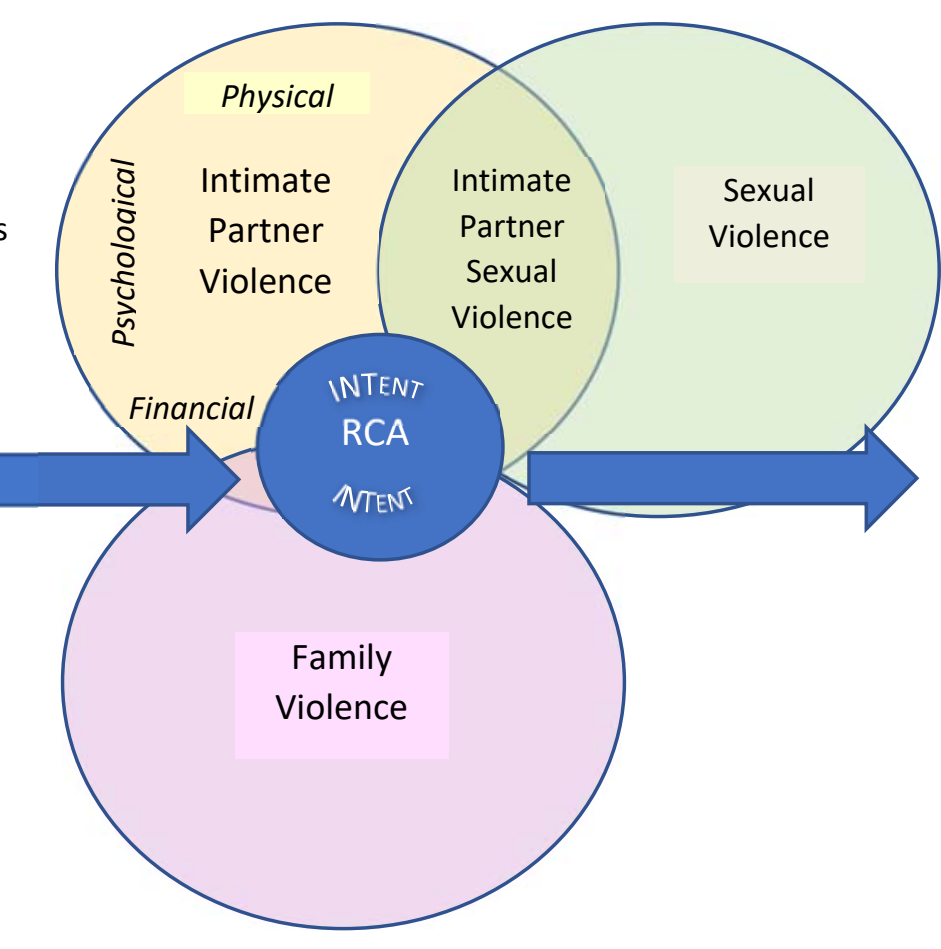

Protective factors

- Government policies that support access to abortion and promote women's rights

- Freely available abortions in a range of settings and locations

- Access to contraception

- Social attitudes that destigmatise abortion, and support women's choices around reproduction and motherhood

- Sexual health education

- Community awareness around RCA

- Gender equality

- Policies promoting equal access to structural supports

Fig.1 Intersections between reproductive coercion and abuse, intimate partner violence, family violence and sexual violence (Tarzia et al. 2018) 
In the specific case of migrant minority ethnic women, a visual representation of how reproductive coercion and abuse is experienced might look something like this (see Figure 2). Figure 2 shows how both structural inequalities and patriarchal

community norms can be harnessed by perpetrators through the use of physical, psychological or sexual violence to control women's reproductive autonomy.

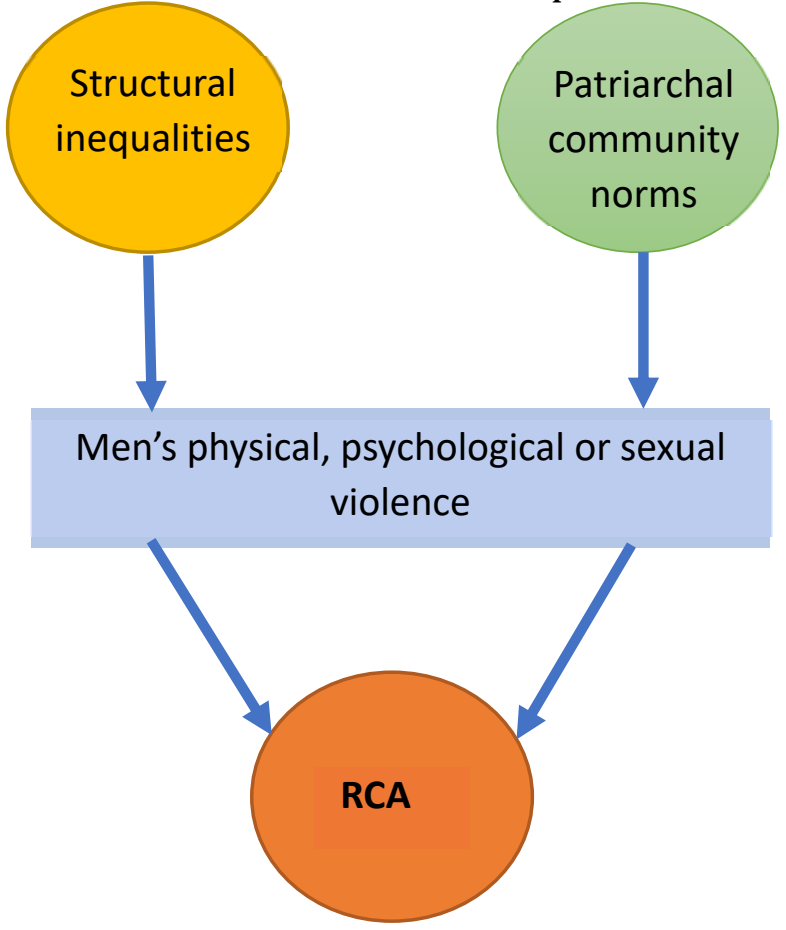

Fig. 2 Migrant minority ethnic women's experience of reproductive coercion and abuse

The findings of the study have important implications for several areas of service provision. First, we suggest that discourse around intimate partner violence and immigration needs to be broadened to include reproductive coercion and abuse as a specific aspect of immigration abuse. Second, health services providing antenatal or abortion care to migrant minority ethnic women ought to be knowledgeable about the possibility of reproductive coercion and abuse and be alert to possible risk factors such as insecure visa status. It is critical that migrant minority ethnic women be able to access financial support to reduce the likelihood that they will be forced into an unwanted pregnancy or forced to terminate a wanted one. Lastly, as others have advocated (Ussher et al. 2017), it is essential for migrant minority ethnic women to be provided with culturally appropriate education around contraception, including options that are undetectable by the perpetrator.

\section{Limitations}

Although this study provides an important first glimpse into the issues faced by migrant minority ethnic women in Australia, it has a number of limitations. First, we explored only the views of service providers. Thus, the voices of migrant minority ethnic women themselves are not present. Future research must ensure engagement with survivors to avoid marginalising them further. Second, given that we worked with specialist women's 
services, the participants were mostly only seeing women who had sought support for violence in their relationship. Thus, the potential role of culture as a protective factor against reproductive coercion and abuse was not present in the data. Third, none of the authors come from migrant or refugee backgrounds. This may have led us to approach the topic in a way that reflects our position of privilege. Lastly, we acknowledge that migrant minority ethnic women are diverse; and that attitudes towards sex and reproduction vary enormously across and within communities. As stated earlier, it is not our intention to make generalising statements about "culture" as a homogenous entity, but rather, to suggest further areas for exploration with different migrant communities to examine these experiences further.

\section{Conclusion}

This paper opens up a trajectory for further research with migrant minority ethnic women. Our findings confirm assertions by Tarzia and colleagues (2018) that there is a complex interplay between reproductive coercion and abuse, sexual violence and intimate partner violence, but highlight ways in which the additional impact of structural issues and patriarchal gender norms within some communities increases the level of risk for migrant minority ethnic women. More research specifically focusing on reproductive coercion and abuse in minority ethnic populations is needed in order to further understand the dynamics of this hidden form of violence and how it may vary across and within communities.

\section{Acknowledgements}

We acknowledge all the service providers who gave up their time to participate in this research.

\section{Declaration of Interest}

The authors have no competing interests to declare. 


\section{References}

Abraham, M. 1999. "Sexual Abuse in South Asian Immigrant Marriages." Violence Against Women 5 (6): 591-618.

Ahmad, F., N. Driver, M. Mcnally, and D. Stewart. 2009. ""Why Doesn't She Seek Help for Partner Abuse?" An Exporatory Study with South Asian Immigrant Women." Social Science \&Medicine 2009 6: 613-22.

Ahmad, F., S. Riaz, P. Barata, and D. Stewart. 2004. "Patriarchal Beliefs and Perceptions of Abuse among South Asian Immigrant Women." Violence Against Women 10 (3): 262-82.

Allimant, A., and B. Ostapiej-Piatkowski. 2011. "Supporting Women from CALD Backgrounds Who Are Victim/Survivors of Sexual Violence: Challenges and Opportunities (ACSSA Wrap No.9)." Melbourne: Australian Institute of Family Studies.

Allotey, P., L. Manderson, S. Baho, and L. Demian. 2004. "Reproductive Health for Resettling Refugee and Migrant Women." Health Issues 78 (2004): 12-7.

AMES Australia, and Australian Government Department of Social Services. 2016. "Violence against Women in CALD Communities: Understandings and Actions to Prevent Violence against Women in CALD Communities." Canberra: AMES Australia \& Commonwealth Department of Social Services.

Australian Bureau of Statistics. 2016. "2016 Census: Multicultural Centus Reveals a Fast Changing, Culturally Diverse Nation."

- - . Migration, Australia, 2018-19, Cat. No. 3412.0." ABS. https://www.abs.gov.au/ausstats/abs@.nsf/mf/3412.0/.

Ayyub, R. 2000. "Domestic Violence in the South Asian Muslim Immigrant Population in the United States." Journal of Social Distress \& the Homeless 9 (3): 237-48.

Balice, G., S. Aquino, S. Baer, M. Behar, A. Belur, J. Filitter, T. Howard, et al. 2019. "A Review of Barriers to Treating Domestic Violence for Middle Eastern Women Living in the United States." Psychology and Cognitive Sciences Open Journal 5 (1): 30-6.

Basile, K. C., S. Smith, Y. Liu, M. Kresnow, A. Fasula, L. Gilbert, and J. Chen. 2018. "Rape-Related Pregnancy and Association with Reproductive Coercion in the U.S." American Journal of Preventive Medicine 55 (6):770-6. doi: 10.1016/j.amepre.2018.07.028.

Braun, V., and V. Clarke. 2019. "Reflecting on Reflexive Thematic Analysis." Qualitative Research in Sport, Exercise and Health 11 (4): 589-97. doi: https://doi.org/10.1080/2159676X.2019.1628806.

Brownridge, D. A., T. L. Taillieu, K. A. Tyler, A. Tiwari, K. L. Chan, and S. C. Santos. 2011. "Pregnancy and Intimate Partner Violence: Risk Factors, Severity, and Health Effects." Violence Against Women 17 (7): 858-81. doi: 10.1177/1077801211412547.

Cappelletti, M., J. Gatimu, and G. Shaw. "Exposing Reproductive Coercion: A Toolkit for Awareness Raising, Assessment and Intervention." Feminist Women's Health Centre, National Coalition Against Domestic Violence,

National Organization for Men Against Sexism, Accessed 8/7/20. https://www.communitysolutionsva.org/files/Reproductive-Health-IPV_Wheel.pdf. 
Childress, S. 2013. "A Meta-Summary of Qualitative Findings on the Lived Experience among Culturally Diverse Domestic Violence Survivors." Issues in Mental Health Nursing 34 (9): 693-705.

Do, K., B. Weiss, and A. Pollack. 2013. "Cultural Beliefs, Intimate Partner Violence, and Mental Health Functioning among Vietnamese Women." International Perspectives in Psychology: Research, Practice, Consultation 2 (3): 149-63.

Douglas, H., and K. Kerr. 2018. "Domestic and Family Violence, Reproductive Coercion and the Role for Law." Journal of Law and Medicine 26 (2):341-55.

Edvardsson, K., A. Axmon, R. Powell, and M. Davey. 2018. "Male-Biased Sex Ratios in Australian Migrant Populations: A Population-Based Study of 1191350 Births 1999-2015." International Journal of Epidemiology 47 (6): 2025-37.

Fakunmoju, S., and F. Bammeke. 2017. "Gender-Based Violence Beliefs and Stereotypes: CrossCultural Comparison across Three Countries." International Journal of Asian Social Science 7 (9): 738-53.

Flood, M. 2015. "Work with Men to End Violence against Women: A Critical Stocktake." Culture, Health \& Sexuality 17 (sup2): 159-76.

Forbes-Mewett, H., and J. Mcculloch. 2015. "International Students and Gender-Based Violence." Violence Against Women 22 (3): 344-65. doi: 10.1177/1077801215602344.

Grace, K., M. Decker, K. Alexander, J. Campbell, E. Miller, N. Perrin, and N. Glass. 2020. "Reproductive Coercion, Intimate Partner Violence, and Unintended Pregnancy among Latina Women." Journal of Interpersonal Violence Online first. https://doi.org/10.1177/0886260520922363

Grace, K. T., and J. C. Anderson. 2018. "Reproductive Coercion: A Systematic Review." Trauma Violence \& Abuse 19 (4): 371-90.

Hawkey, A., J. Ussher, and J. Perz. 2018. "'If You Don't Have a Baby, You Can't Be in Our Culture": Migrant and Refugee Women's Experiences and Constructions of Fertility and Fertility Control." Women's Reproductive Health 5 (2): 75-98.

Kasturirangan, A., S. Krishnan, and S. Riger. 2004. "The Impact of Culture and Minority Status on Women's Experience of Domestic Violence." Trauma, Violence and Abuse 5 (4):318-32.

Katz, J., E. Poleshuck, B. Beach, and R. Olin. 2017. "Reproductive Coercion by Male Sexual Partners: Associations with Partner Violence and College Women's Sexual Health." Journal of Interpersonal Violence 32 (21): 3301-20.

Kazmerski, T., H. Mccauley, K. Jones, S. Borrero, J. Silverman, M. Decker, D. Tancredi, and E. Miller. 2015. "Use of Reproductive and Sexual Health Services among Female Family Planning Clinic Clients Exposed to Partner Violence and Reproductive Coercion." Maternal \& Child Health Journal 19 (7): 1490-6. doi: 10.1007/s10995-014-1653-2.

Khoei, E., A. Whelan, and J. Cohen. 2008. "Sharing Beliefs: What Sexuality Means to Muslim Iranian Women Living in Australia." Culture, Health \& Sexuality 10 (3): 237-48.

Lykke, N. 2010. Feminist Studies: A Guide to Intersectional Theory, Methodology and Writing. New York: Routledge.

Maher, J., and M. Segrave. 2018. "Family Violence Risk, Migration Status and 'Vulnerability': Hearing the Voices of Immigrant Women." Journal of Gender-Based Violence 2 (3): 50318. 
Mccauley, H., K. Falb, T. Streich-Tilles, D. Kpebo, and J. Gupta. 2014. "Mental Health Impacts of Reproductive Coercion among Women in Cote D'ivoire." International Journal of Gynaecology \& Obstetrics 127 (1): 55-9.

Mengesha, Z., J. Perz, T. Dune, and J. Ussher. 2017. "Refugee and Migrant Women's Engagement with Sexual and Reproductive Health Care in Australia: A Socio-Ecological Analysis of Health Care Professional Perspectives." PLOS ONE 12: e0181421. doi: 10.1371/journal.pone.0181421.

Miller, E., M. Decker, H. Mccauley, D. Tancredi, R. Levenson, J. Waldman, P. Schoenwald, and J. Silverman. 2010. "Pregnancy Coercion, Intimate Partner Violence and Unintended Pregnancy." Contraception 81 (4):316-22. doi: 10.1016/j.contraception.2009.12.004.

Nikolajski, C., E. Miller, H. L. Mccauley, A. Akers, E. Schwarz, and L. Freedman. 2015. "Race and Reproductive Coercion: A Qualitative Assessment." Women's Health Issues 25 (3): 21623.

Price, E., L. Sharman, H. Douglas, N. Sheeran, and G. Dingle. 2019. "Experiences of Reproductive Coercion in Queensland Women." Journal of Interpersonal Violence Online first:1-21. doi: $10.1177 / 0886260519846851$.

Raj, A., and J. Silverman. 2002. "Violence against Immigrant Women: The Roles of Culture, Context and Legal Immigrant Status on Intimate Partner Violence." Violence Against Women 8 (3): 367-98.

Raj, A., J. Silverman, J. Mccleary-Sills, and Liu R. 2005. "Immigration Policies Increase South Asian Immigrant Women's Vulnerability to Intimate Partner Violence." Journal of the American Medical Women's Association 60 (1): 26-32.

Rowlands, S., and S. Walker. 2019. "Reproductive Control by Others: Means, Perpetrators and Effects." BMJ Sexual and Reproductive Health 45: 61-7.

Segrave, M. 2017. "Temporary Migration and Family Violence: An Analysis of Victimisation, Vulnerability and Support." Melbourne: School of Social Sciences, Monash University.

Sharman, L., H. Douglas, E. Price, N. Sheeran, and G. Dingle. 2018. "Associations between Unintended Pregnancy, Domestic Violence, and Sexual Assault in a Population of Queensland Women." Psychiatry, Psychology and Law 26 (4): 541-52.

Tarzia, L. 2020. "Toward an Ecological Understanding of Intimate Partner Sexual Violence." Journal of Interpersonal Violence Online first. https://doi.org/10.1177/0886260519900298.

Tarzia, L., M. Wellington, J. Marino, and K. Hegarty. 2018. "'A Huge, Hidden Problem': Australian Health Practitioners' Views and Understandings of Reproductive Coercion." Qualitative Health Research Online first. doi: https://doi.org/10.1177/1049732318819839.

Ussher, J., J. Perz, C. Metesula, A. Hawkey, M. Morrow, R. Narchal, and J. Estoesta. 2017. "Negotiating Discourses of Shame, Secrecy, and Silence: Migrant and Refugee Women's Experiences of Sexual Embodiment." Archives of Sexual Behavior 46: 1901-21.

Ussher, J., M. Rhyder-Obid, J. Perz, M. Rae, T. Wong, and P. Newman. 2012. "Purity, Privacy and Procreation: Constructions and Experiences of Sexual and Reproductive Health in Assyrian and Karen Women Living in Australia." Sexuality and Culture 16 (467-485).

Vaughan, C., E. Davis, A. Murdolo, J. Chen, L. Murray, R. Quiazon, K. Block, and D. Warr. 2016. "Promoting Community-Led Responses to Violence against Immigrant and Refugee 
Women in Metropolitan and Regional Australia: The Aspire Project: Resesearch Report (Anrows Horizons 07/2016)." Sydney, NSW: ANROWS.

Webster, K., K. Diemer, N. Honey, S. Mannix, J. Mickle, J. Morgan, A. Parkes, et al. 2018. "Australians' Attitudes to Violence against Women and Gender Equality. Findings from the 2017 National Community Attitudes Survey (NCAS)." Sydney NSW: ANROWS. 